\title{
Synthesis and Properties of Adhesive Polymer-Methylmethacrylate Materials
}

\author{
Ulyana Khromiak (DD, ${ }^{1}$ Volodymyr Levytskyi, ${ }^{2}$ Kateryna Stepova, ${ }^{1}$ and Andry Tarnawsky \\ ${ }^{1}$ Department of Environmental Safety, Lviv State University of Life Safety, 35 Kleparivska Str., Lviv 79000, Ukraine \\ ${ }^{2}$ Department of Chemical Technology of Plastics Processing, Lviv Polytechnic National University, \\ 12 S. Bandery Str., Lviv 79013, Ukraine
}

Correspondence should be addressed to Ulyana Khromiak; ulanajukovska@gmail.com

Received 23 October 2017; Revised 2 February 2018; Accepted 21 February 2018; Published 27 March 2018

Academic Editor: Marta Fernández-García

\begin{abstract}
Copyright ( 2018 Ulyana Khromiak et al. This is an open access article distributed under the Creative Commons Attribution License, which permits unrestricted use, distribution, and reproduction in any medium, provided the original work is properly cited.
\end{abstract}

\begin{abstract}
Kinetics of emulsion polymerization of hydrophilic vinyl monomers in the presence of polyvinylpyrrolidone and technological principles of their synthesis are determined. Reasonable technological parameters in the synthesis of copolymers are determined. Physicochemical properties of the synthesized copolymers (surface tension, the size of latex particles, and $\mathrm{pH}$ ) are determined. Synthesized graft copolymers were used to create high-adhesion polymer-monomer compositions. These compositions have high reactivity at room temperature. It can be regulated by the nature of the polymer matrix and the introduction of comonomers and fillers due to the influence of physicochemical factors on the process of polymer formation. The rate of polymerization and the degree of monomer conversion largely depend on the nature of the polymer matrix. The highest polymerization rate and the maximum degree of conversion are observed when using a copolymer of polyvinylpyrrolidone and polymethylmethacrylate. Materials based on the developed compositions are characterized by a low residual monomer content and high operational properties, such as surface hardness, Vicat softening temperature, and adhesive bond strength to supports of different nature.
\end{abstract}

\section{Introduction}

The contemporary development of science and technology requires the creation of new polymer materials with a corresponding set of special properties: high adhesion to supports of different nature, high physicomechanical, thermophysical, and selective-sorption properties. These include low-toxic adhesive polymer-monomer compositions of medical and general technical purposes.

Among the methods for preparation of such materials with the necessary properties the most interesting are those based on the modification of known industrial polymers in polymerization processes. These methods make it possible to obtain polymer materials with the preferable set of technological and operational properties at rather low material and energy costs.

The determining factor for most of the technologies based on the preparation of modified polymeric materials is modifier. Polymers with high surface activity, good solubility in water and many aqueous organic media, high ability to complex formation, and high sorption characteristics are used as modifiers $[1,2]$.

Polyvinylpyrrolidone (PVP) and its copolymers belong to such polymers. Due to their specific properties, additional opportunities for improving modern technologies [3, 4], obtaining of new functionalized materials [5], and expanding the branches of their use are opened.

Monomer-polymer compositions are widely used in a variety of industries especially as a basis for preparing materials with enhanced adhesion properties. Among such materials, acrylates based adhesives occupy a special place [6]. First of all, the increased interest in acrylates-containing glues is caused by their high operational properties: cure speed, adhesion to a variety of materials, strength and durability of adhesive compounds, and their low toxicity [7]. In this regard, these materials are widely used in a variety of industries, namely, in medicine, dentistry, construction, engineering, automotive, and electrical and textile industries [8]. It should 
be noted that the use of (meth)acrylic adhesives is expanding in current conditions to solve many technological tasks [9].

The main components of (meth)acrylic adhesives are (meth)acrylic monomers or polymers, initiating systems, hardeners, stabilizers, and modifiers. Methylmethacrylate (MMA) is often used as the main monomer in the (meth)acrylic adhesives. It provides high adhesive strength and is a good solvent for a number of polymers. When combined with oils MMA makes it possible to glue untreated surfaces. MMA has several disadvantages, in particular, high volatility, pungent odor, and fire risk. It is used in admixture with high boiling monomers such as hydroxyalkyl methacrylate, glycidyl methacrylate, and methacrylates of higher alcohols: tetrahydrofurfuryl-, diethylaminoethyl-, 2oxopropyl neopentyl-, and other glycolmethacrylates and styrene [10-12].

The aim of the work is the development of functionalized materials based on graft copolymers of polyvinylpyrrolidone for high-adhesion methyl methacrylate-copolymer compositions.

\section{Materials and Methods}

Copolymers were synthesized by emulsion polymerization of vinyl monomers in the presence of PVP, potassium persulfate, or hydrogen peroxide at $60-70^{\circ} \mathrm{C}$ and the ratio of monomer: aqueous phase $=1: 3$.

The kinetics of the emulsion polymerization of the test compositions were studied by dilatometric method for measuring the volume change of the reaction mixture during polymerization.

IR studies were carried out on a spectrograph "Specord75IR" at room temperature. The spectra were recorded at the same intensity and the same scan rate between 400 and $4000 \mathrm{~cm}^{-1}$.

The adhesive compositions were synthesized by the polymerization of MMA or a mixture thereof with other monomers in the presence of synthesized copolymers at room temperature. To determine the properties of the adhesive compositions, a number of studies were carried out. The conversion degree of the monomer was determined by the bromide-bromate method. The surface hardness and Vicat softening temperature of the samples were measured on the Heppler consistometer. Adhesion was determined according to ISO 527-5: 2009.

\section{Results and Discussion}

3.1. Technological and Kinetic Features of Emulsion Polymerization of Vinyl Monomers in the Presence of PVP. It has been established that the enhanced initiating ability of the series of (meth) acrylic monomers has graft copolymers of PVP. Copolymers were synthesized on the basis of PVP and vinyl monomers (MMA, vinyl acetate (VA), and styrene) by emulsion polymerization. The emulsion polymerization of vinyl monomers is mostly carried out in the presence of emulsifiers that are surfactants of different nature [1315]. The nature of the emulsifier and its concentration in the reaction medium greatly influence the mechanism and kinetics of the polymerization and therefore the technological features of the process and its productivity. This influence is caused by changes in the interphase characteristics of the polymerization system, adsorption phenomena at the phase boundary, and changes in the solubility of monomers in the dispersion medium with the active participation of the emulsifier. Emulsifiers have to meet a number of requirements $[13,14]$ : high surface activity, ability to micellization, and nontoxicity.

Due to its properties and structure PVP can serve as an emulsifier in the processes of emulsion polymerization and also be an effective polymer matrix, which actively participates directly in the polymerization processes. It should be noted that the influence of the emulsifier on the regularities of the emulsion polymerization of vinyl monomers primarily resulted in change in the interphase characteristics of the polymerization system and the solubility of the monomers in the reaction medium. On the basis of the conducted studies, it has been established that PVP macromolecules exhibit high surface activity on the interphase surface of watervinyl monomer. This activity increases with a decrease in the polarity of the monomer in the VA-MMA-styrene series. This is evidenced by the nature of the interphase tension isotherms in the vinyl monomer-aqueous PVP solution system, the PVP adsorption dependencies in the coordinates of the Langmuir equation, the maximum values of the Gibbs adsorption $\left(G_{m}=\right.$ $\left.2.14 \cdot 10^{-6} \mathrm{~mol} / \mathrm{m}^{2}\right)$, and adsorption work $\left(W_{\mathrm{ad}}=48.8\right.$. $10^{-3} \mathrm{~J} / \mathrm{m}^{2}$ ) for styrene. PVP has different effects on the total (true and colloidal) solubility of vinyl monomers in aqueous solution.

Due to the increase of the PVP concentration up to $0.2 \mathrm{~mol} / \mathrm{l}$ monomer concentration, solubility of MMA increases from 0.15 to $0.22 \mathrm{~mol} / \mathrm{l}$, for VA it decreases from 0.33 to 0.18 , and for styrene it is almost unchangeable. Obviously it is a consequence of specific intermolecular interactions in the system that will affect the polymerization processes in these systems.

High surface activity and solubilization ability of PVP prove its high efficiency as an emulsifier in the processes of emulsion polymerization of vinyl monomers (KKM PVP, 0.09-0.11 mole/liter monomer concentration).

The effect of the monomer nature (MMA and styrene), concentration factors, temperature, and stirring rate on the kinetic regularities the of emulsion polymerization process of vinyl monomers in the presence of PVP was established. Emulsion polymerization of vinyl monomers in the presence of PVP is characterized by a different rate depending on the nature of the monomer (Figure 1).

Obviously the features of the emulsion polymerization of vinyl monomers are caused by the dependence of the mechanism of particle formation from the solubility of the monomer in the aqueous solution of PVP: during the MMA polymerization the process may run in the aqueous solution with the formation of macroradicals by homogeneous nucleation, as well as in the micelles of the emulsifier (PVP), and continues in the polymer-monomeric particles, which are formed from the emulsifier micelle or from aggregates of macromolecules released from the water phase. In the case of styrene, the polymerization process begins in the micelles 


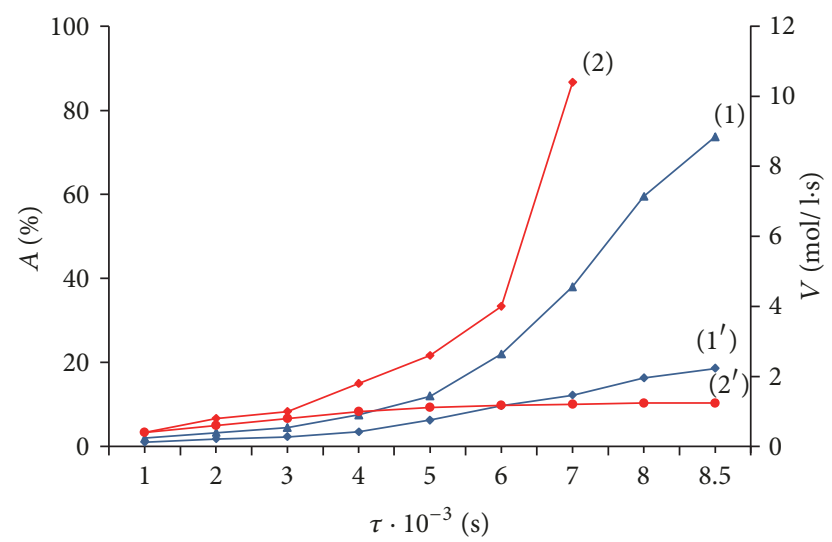

Figure 1: Degree and rate of emulsion polymerization of vinyl monomers in the presence of PVP: (1), $\left(1^{\prime}\right)-\mathrm{A} ;(2),\left(2^{\prime}\right)-V$. (1), (2)MMA. $\left(1^{\prime}\right),\left(2^{\prime}\right)$ styrene. $C_{\text {mon. }}=0.99 \mathrm{~mol} / \mathrm{l} ; C_{\text {in. }}=2.81 \cdot 10^{-3} \mathrm{~mol} / \mathrm{l}$; $C_{\mathrm{PVP}}=0,09 \mathrm{~mol} / \mathrm{l} ; M_{\mathrm{PVP}}=28000 ; T=323 \mathrm{~K}$.

of the emulsifier and continues in the polymer-monomer particles formed from them. In addition, polymerization of styrene can occur in micelles and in microdrops of a monomer, which is specific for nonionic emulsifiers (like PVP).

The polymerization reaction rates for the monomer and initiator are 1.9 and 0.75 , respectively, for MMA and 1.6 and 0.55 for styrene.

It was found that the polymerization rate of all the investigated vinyl monomers increases with increase in ratio of the monomeric and aqueous phases, the concentration of the initiator, and the temperature. Dependence of the MMA polymerization rate on the particle size in the range of $70-200 \mathrm{~nm}$ is described by the following:

$$
V_{\text {pol.MMA }}=1 \cdot 10^{-4} \cdot r^{2}-0.055 \cdot r+5.603 .
$$

As a result of polymerization, graft copolymers of PVP are formed in the investigated systems. This is evidenced by the physicochemical and IR spectroscopic studies. The IR spectra of synthesized methylmethacrylic copolymers (Figure 2) have characteristic bands that are related to both the PVP links $\left(1300 \mathrm{~cm}^{-1}, 1450 \mathrm{~cm}^{-1}, 1470 \mathrm{~cm}^{-1}\right.$, and $\left.1500 \mathrm{~cm}^{-1}\right)$ and PMMA $\left(1140 \mathrm{~cm}^{-1}, 1160 \mathrm{~cm}^{-1}, 1180 \mathrm{~cm}^{-1}, 1200 \mathrm{~cm}^{-1}\right.$, and $1220 \mathrm{~cm}^{-1}$ ).

The degree and effectiveness of grafting depend significantly on the conditions of the process, in particular the PVP concentration in the aqueous medium and the temperature of the process.

It has been established that the properties of latexes obtained by emulsion polymerization (surface tension, $\mathrm{pH}$, and particle radius) depend on the conditions of the process: the ratio of the monomeric and aqueous phases, the temperature, the concentration of the monomer and initiator, and the amount of PVP. As the concentration of the initiator increases and the temperature of the process decreases, the size of the latex particles and the $\mathrm{pH}$ decrease (Figure 3).

The ratio between the components of the polymerization system and the mixing rate also has a significant effect on

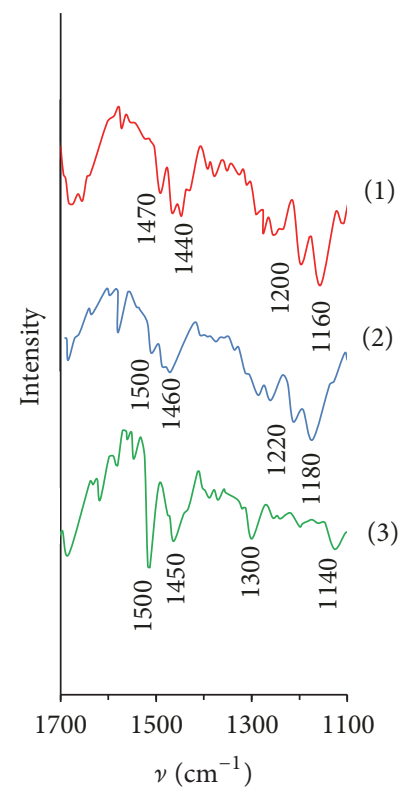

FIGURE 2: IR spectra of PVP-gr-PMMA copolymers depending on the ratio of components in the synthesis and the drying temperature of the copolymer: MMA: PVP in the synthesis of the copolymer, mol/mol monomer concentration: (1), (2) $3: 1$; (3) $1: 1 . T_{d}, K:(1)$ 313; (2), (3) 343.

the size of latex particles. The size of the latex particles also depends on the nature of the monomer and, depending on the mixing rate, is described by the following:

for styrene: $r_{\mathrm{PVP}-\mathrm{gr}-\mathrm{PS}}$

$$
\begin{aligned}
= & -2 \cdot 10^{-4} \cdot V_{\text {mix. }}^{3}+0.0927 \cdot V_{\text {mix. }}^{2}-15.758 \cdot V_{\text {mix. }} \\
& +988.58,
\end{aligned}
$$

for MMA: $r_{\text {PVP-gr-PMMA }}$

$$
\begin{aligned}
= & -9 \cdot 10^{-5} \cdot V_{\text {mix. }}^{3}+0.0517 \cdot V_{\text {mix. }}^{2}-9.6168 \cdot V_{\text {mix. }} \\
& +666.24 .
\end{aligned}
$$

In our opinion, an increase in the particle radius and, correspondingly, a decrease in their total quantity, with an increase in the initial concentration of the monomer, can be attributed to the increase in the viscosity of the polymerization system, which leads to a decrease in the mobility of the radicals formed during the decomposition of the potassium persulfate and, as a consequence, a decrease in the initial quantity of polymer-monomer particles. Also, it should be noted that, according to the classical theory of emulsion polymerization, when the conversion rate reaches $10-15 \%$, the rate increases due to a termination constant decrease. This leads to an increase of particles size of synthesized latex and changes in their structure and properties.

Synthesized PVP copolymers have sufficiently high sorption properties that can be controlled by polymerization conditions, primarily by the process temperature and the concentration of the initiator. As the polymerization temperature 


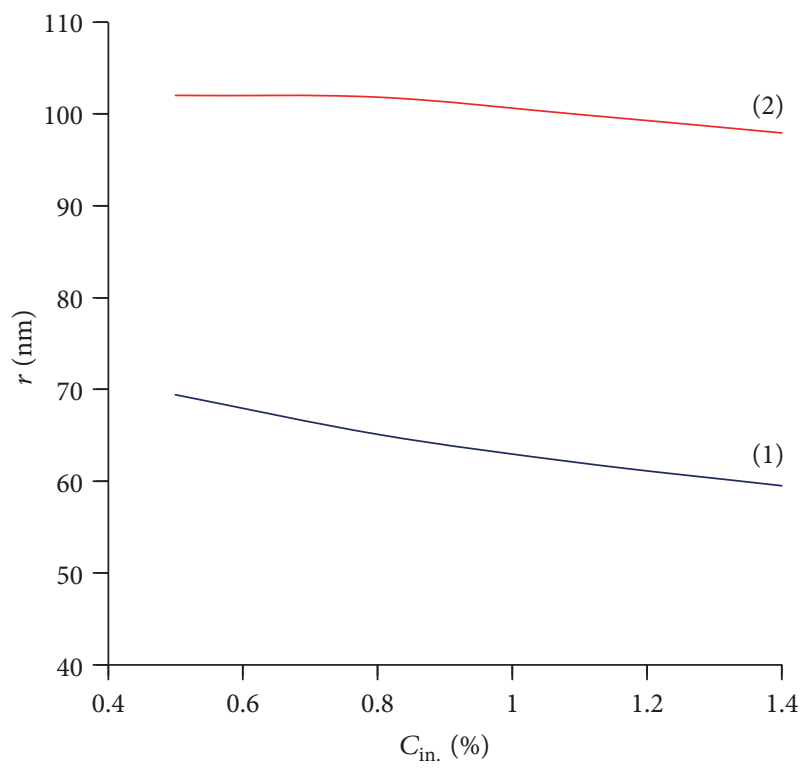

(a)

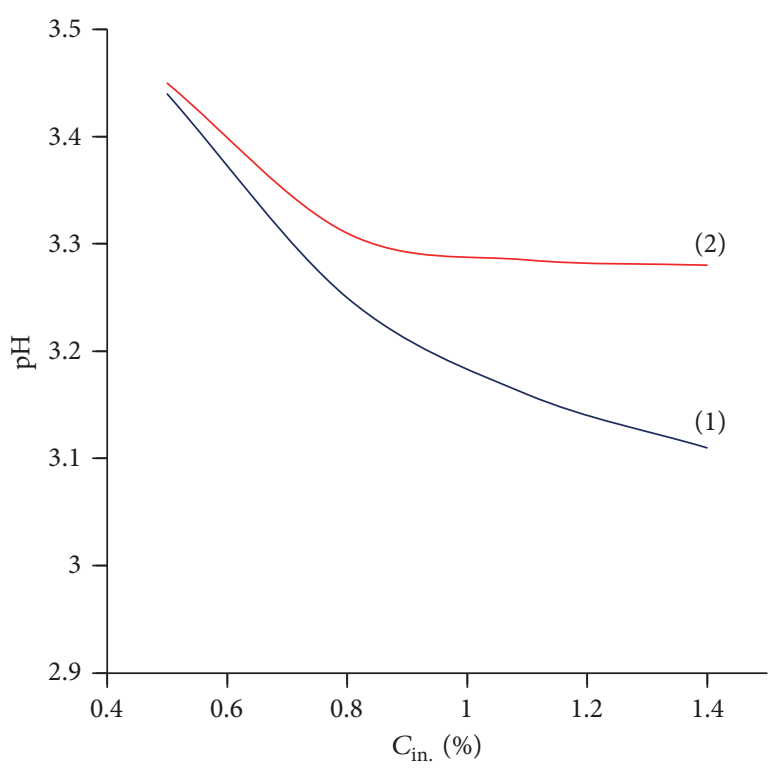

(b)

FIGURE 3: Influence of synthesis conditions on the properties of copolymer latexes: (a) r; (b) pH. (1) $323 \mathrm{~K}$; (2) $333 \mathrm{~K}$.

increases and the initiator concentration decreases, the sorption capacity of the copolymers decreases. Sorption ability of finely dispersed graft copolymers of polyvinylpyrrolidone and polystyrene (PVP-gr-PS) is greater, compared to the graft copolymer of polyvinylpyrrolidone and polymethylmethacrylate (PVP-gr-PMMA). With respect to heparin, it is $3.0 \cdot 10^{5}$ and $3.1 \cdot 10^{4}$ units $/ \mathrm{m}^{2}$, respectively, with respect to iodine 36 and $32 \mathrm{~mol} / \mathrm{g}$. It can be used in the preparation of sorption materials for antiseptics, therapeutic insulating films, and thrombus-resistant products.

3.2. Discussion of the Results of the Investigation of the Technological and Physicochemical Basis for the Development of Methyl Methacrylate-Copolymer Compositions and Materials Based on Them. The emulsion polymerized graft copolymers of PVP were used as a polymer matrix for the preparation of MMA-copolymer compositions cured by block polymerization.

The results of kinetic studies of MMA polymerization in the presence of finely dispersed PVP copolymers depending on the nature of the polymer matrix (PM) and the conditions for its synthesis are shown in Figure 4.

The rate of polymerization and the degree of monomer conversion largely depend on the nature of the polymer matrix (Figure 4). Polymerization of MMA occurs at a high rate and, depending on the nature of the polymer matrix, the monomer conversion rate of $70-90 \%$ is achieved in $10-15$ minutes. The highest polymerization rate and the maximum degree of conversion are observed when using the PVP-grPMMA copolymer.

It was found that the activity of the polymer matrix also depends on the conditions of its synthesis. A polymer matrix synthesized with a smaller amount of PVP exhibits significantly more activity during block polymerization of MMA. This, along with other factors, is a consequence of the

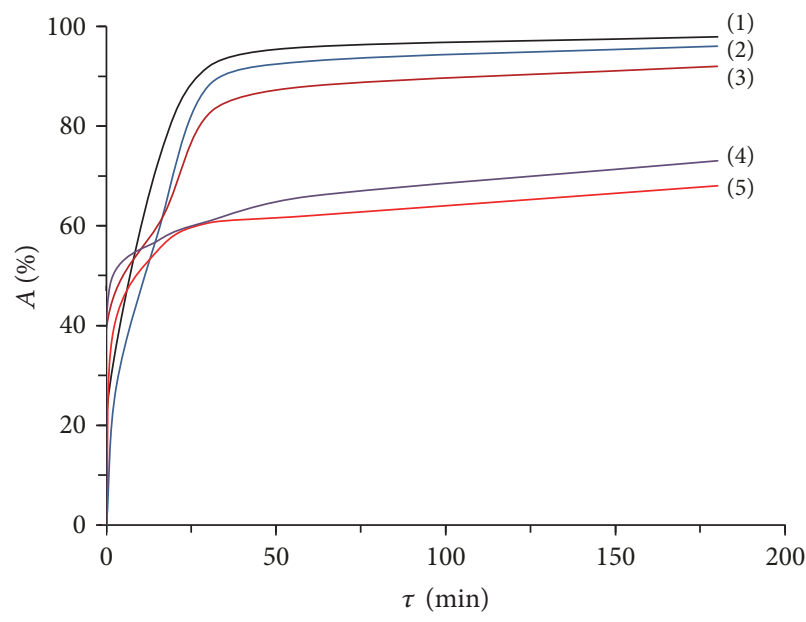

FIGURE 4: Kinetic curves of polymerization of MMA compositions depending on the nature and conditions of PM synthesis: (1), (2), (4) PVP-gr-PMMA; (3) PVP-gr-PS; (5) PMMA. The ratio of MMA : PVP in the synthesis of PM, mole/mole monomer concentration: (1)-(3) $3: 1$; (4) $1: 1$. Td. PM, K: (1), (3), (5) 313; (2), (4) 343.

greater affinity of the monomeric and polymeric phases. It should be noted that the drying temperature of the polymer matrix also affects its activity. The copolymers dried under milder conditions ( $313 \mathrm{~K}$ ) show greater activity.

In our opinion, the high polymerization rate of methyl methacrylate can be explained by intermolecular interactions between the components of the reaction medium, which may lead to the formation of a charge transfer complex (CTC) between a double bond of a monomer and a carbamate group of PVP and higher affinity between the monomeric and polymeric phases. Interactions of this nature lead to a redistribution of electronic density on a dual bond of 


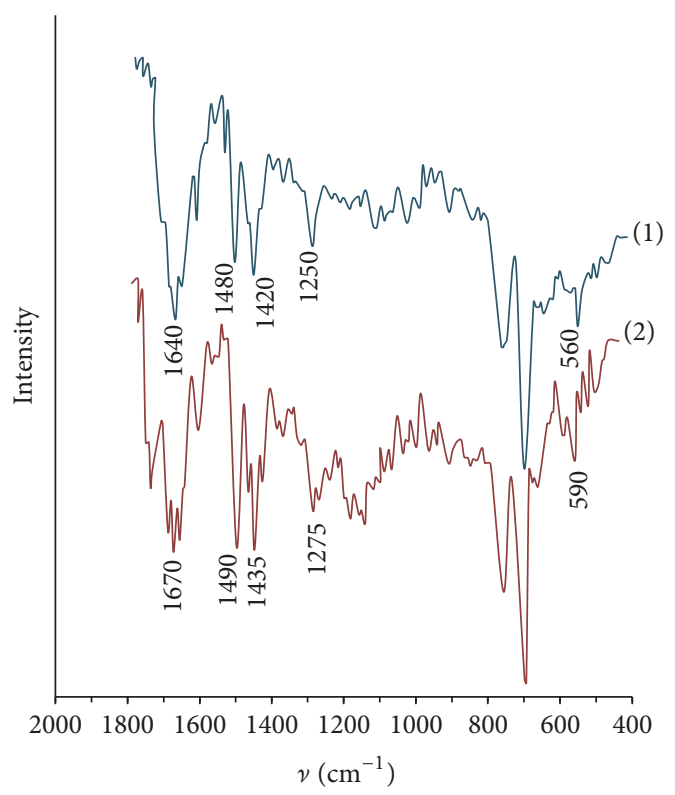

FIGURE 5: IR spectra of copolymer PVP-gr-PS (1) and MMA, a copolymer composition on based on it (2).

monomer and, consequently, to a decrease in the total activation energy of the polymerization process.

The results of IR-spectroscopy studies prove the intermolecular interactions with carbamate groups of PVP in the polymerization system and the morphology of synthesized copolymers and monomer-polymeric materials (Figure 5).

It should be noted that, for the pure PVP, the characteristic absorption bands are 560, 1300, 1415, 1480, and $1735 \mathrm{~cm}^{-1}$. As we see, for copolymer PVP-gr-PS and methylmethacrylate copolymer composition, these bands are shifted.

This displacement is especially apparent for carbamate groups of the pyrrolidone ring and the methylene groups of the macrochain (Table 1).

Obviously, these groups take an active part in the intermolecular interactions between the components of the system. In this case, the activating effect of the polymer matrix on the polymerization process of vinyl monomers is seen. It gives the opportunity to develop compositions that can polymerize under mild conditions without the use of traditional initiators of radical polymerization.

It is established that the kinetic regularities of the polymerization process are affected by the comonomer and mineral filler $\left(\mathrm{Al}_{2} \mathrm{O}_{3}, \mathrm{SiO}_{2}\right)$ (Figure 6).

The addition of comonomers such as 2-hydroxyethylenemethacrylate (HEMA), glycidylmethacrylate (GMA), and vinylpyrrolidone (VP) to MMA leads to a decrease in the polymerization rate, regardless of the nature of the polymer matrix (PVP-gr-PMMA and PVP-gr-PS). It should be noted that the ratio between the monomer and the polymer fines has a significant effect on the polymerization process. The monomer quantity should be as small as possible but enough for complete swelling of the polymer. The swelling rate of the fine powder and the formation of a homogeneous mass essentially depends on the temperature, the molecular

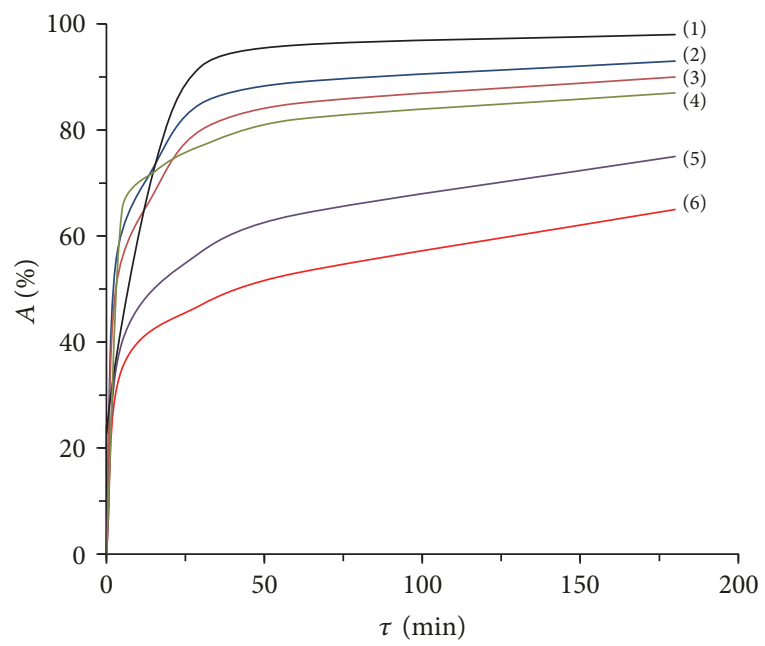

FIGURE 6: Kinetic curves of MMA polymerization in the presence of PVP-gr-PMMA depending on nature and content of comonomer and filler. Comonomer: (2) HEMA, (3) GMA, and (4) VP. Filler: (5) $\mathrm{Al}_{2} \mathrm{O}_{3}$, and (6) $\mathrm{SiO}_{2}$. Comonomer content: $20 \%$ (mas.). Filler content: $10 \%$ (mas).

weight of the polymeric component, the shape and size of the particles, and the monomer:polymer ratio. As it was established on the basis of kinetic studies, when the monomer: copolymer ratio changes from $1: 1.5$ to $1: 3$, time of gel formation decreases about 10 times. The optimal ratio between the monomeric and the polymeric phases for the investigated systems is $1: 2$.

The addition of various fillers, namely, silicon (IV) oxide and aluminum oxide, leads to a decrease in the polymerization rate of the MMA and the limiting degree of polymerization.

Depending on the nature of the polymer matrix and the monomeric phase, the ratio between phases and the presence of filler during polymerization under the investigated conditions, a number of stages can be distinguished in terms of physicochemical properties: sandy, sticky, doughlike, viscous, and fully cured.

Duration of each of these stages for different systems based on the PVP-gr-PMMA copolymer is shown in Figure 7.

The sandy stage begins immediately after mixing the components, and, depending on the temperature, it can last from $30 \mathrm{~s}$ to $5 \mathrm{~min}$. In this case, only the swelling of the finely divided polymer in the monomer begins. It occurs on the surface of the powder particles and depends on their dimensions. In this regard, the polymerization system has no plasticity and the material contains a large amount of unbound monomer. For some systems there is no sand stage. The sticky stage is characterized by the appearance of yarns, sticky mass properties, and great ductility and flowability, with the material having high adhesion. The dough-like stage is characterized by high plasticity, low fluidity, and lack of tackiness. At this stage, the reaction mass can be formed into different products. The rate of transition of the system from the plastic stage to the doughlike one for all polymer matrices is practically the same 


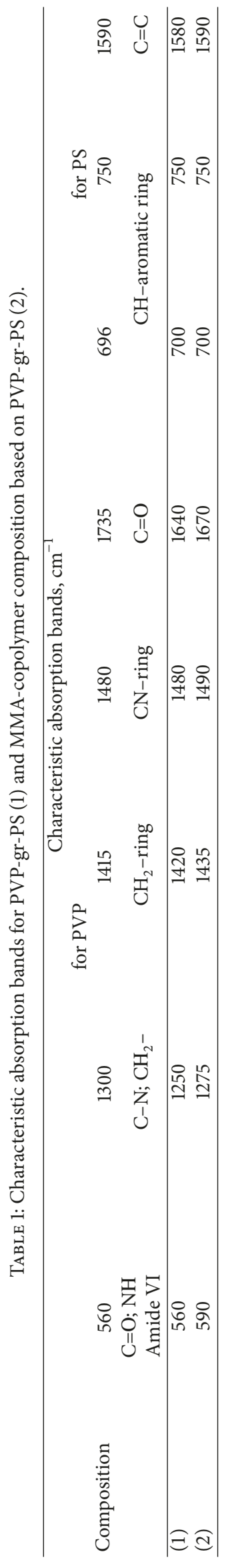




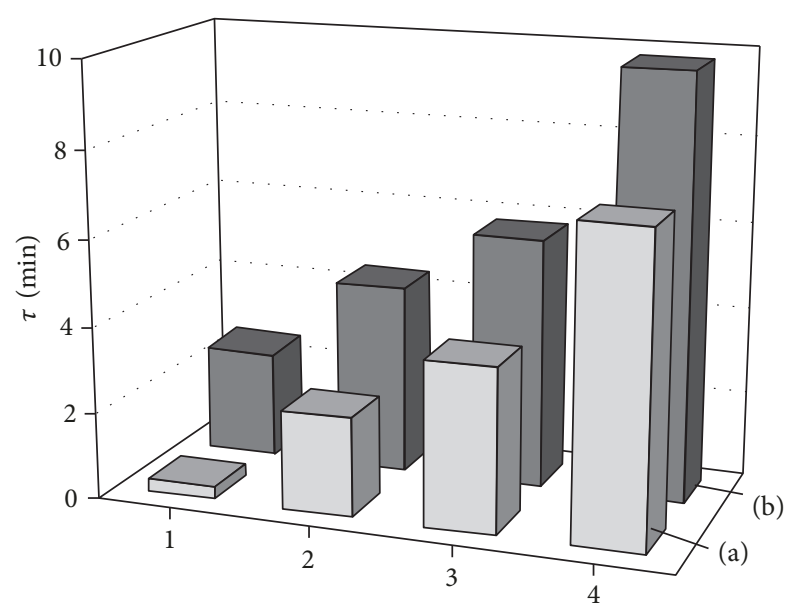

FIgURE 7: Stages of polymerization of MMA-PVP-gr-PMMA compositions depending on comonomer nature: (a) without comonomer; (b) HEMA. Comonomer content: $20 \%$ (mas.). 1: sandy stage; 2: sticky stage; 3: dough-like stage; 4 : viscous stage.

and increases with the increase in their dispersion. The viscous stage is characterized by the preservation of a given shape of the material, even with a short-term mechanical action, and precedes the complete curing of the material. The duration of these stages determines the area of use of the developed compositions: either for glutinous materials, for compounds, or for putty. The presence of this or that stage can be regulated by mixing times and by the ratio of the monomer and polymer phases. All these stages are the result of such chemical and physicochemical processes between the polymeric and monomeric phases as wetting, swelling, dissolution, and polymerization. These stages differ in the different degree of swelling of the polymer matrix, different ductility, fluidity, tackiness, and residual monomer content.

The technological recommendations were developed depending on the further use of the compositions. For glues the mixing time should not be more than 2-2.5 minutes, for putties 3-4 minutes, and for compounds 5-6 minutes. To prevent the formation of air voids in the composition, it is necessary to add the monomer phase to the fine polymer powder in small portions.

The obtained modified materials on the basis of the developed MMA-copolymer compositions have rather high operational properties, such as surface hardness, Vicat softening temperature, and adhesive bond strength to supports of different nature (Figure 8).

It is established that the surface hardness and Vicat softening temperature depend on the nature and conditions of synthesis of the polymer matrix and also on the nature of the comonomer. The addition of bifunctional GMA to the composition results in a significant increase in surface hardness and heat resistance. It may be caused by the formation of more structured materials. Vinylpyrrolidone increases these values, apparently as a result of the formation of a more uniform structure due to the better swelling of the copolymer that contains the PVP units in the monomeric phase. The introduction of comonomers with polar groups into the composition leads to an increase in the strength of the adhesive compound of polymethylmethacrylate materials.

The content of the filler has a significant influence on the surface hardness and Vicat softening temperature. The introduction of $\mathrm{SiO}_{2}$ and the heat treatment at $333 \mathrm{~K}$ lead to an increase in the surface hardness (193 MPa) and Vicat softening temperature $\left(126^{\circ} \mathrm{C}\right)$. Obviously, it happens due to the changes in the submolecular structure of the material caused by chemical and physical factors. Changes in the submolecular structure of the materials caused by heat treatment and the filler confirm the results of the thermomechanical analysis shown in Figure 9.

The decrease of the deformation value in a wide temperature range for thermally treated samples and samples containing the filler is obviously a consequence of increasing the density of the fluctuation network of the polymeric component.

It should be noted that the developed materials have a high enough adhesive strength, regardless of the nature of the substrate. The greatest value of the adhesive strength is observed for PMMA materials, which is caused by their partial swelling due to the MMA diffusion from the monomerpolymer composition. The introduction of comonomers with polar groups into the composition leads to an increase in the adhesive strength of methyl methacrylate materials.

However, process shrinkage $(0.1-1.0 \%)$, water absorption (0.8-1.2\%), thermomechanical properties, and chemical stability in acidic and alkaline media are also dependent on the conditions of synthesis of the polymer matrix and the nature of the comonomer and filler. The materials that contain comonomers such as HEMA and VP, as well as copolymers with a large number of PVP units, exhibit increased water absorption. Obviously, it may be attributed by the fact that due to the presence of carbamate groups PVP has an increased capacity for water sorption, and the presence of hydrophilic HEMA in the monomer phase increases water absorption due to increased nonuniform structure. At the same time, heat treatment as a result of changes in the supramolecular structure leads to a decrease in water absorption. The calculated diffusion coefficients $(7.0 \cdots 7.6$. $\left.10^{-7} \mathrm{sm}^{2} / \mathrm{s}\right)$, sorption coefficients $\left(1.9 \cdots 2.3 \cdot 10^{-2} \mathrm{~g} / \mathrm{sm}^{3}\right)$, and permeability $\left(17.1 \cdots 17.5 \cdot 10^{-9} \mathrm{~g} \cdot \mathrm{sm} /\left(\mathrm{sm}^{2} \cdot \mathrm{s}\right)\right)$ of chemical reagent $\left(\mathrm{NaOH}, \mathrm{H}_{2} \mathrm{SO}_{4}\right)$ in the sample indicate a sufficiently high chemical stability of the developed materials in acidic and alkaline media.

\section{Conclusions}

Monomer-polymer compositions based on MMA and graft copolymers PVP-gr-PMMA or PVP-gr-PS have a high reactivity at room temperature. It can be regulated by the nature of the polymer matrix and the introduction of comonomers and fillers due to the influence of physicochemical factors on the polymerization process.

Materials based on the developed compositions have low content of the residual monomer and sufficiently high operational properties such as surface hardness, heat resistance, and adhesion to supports of different nature. 


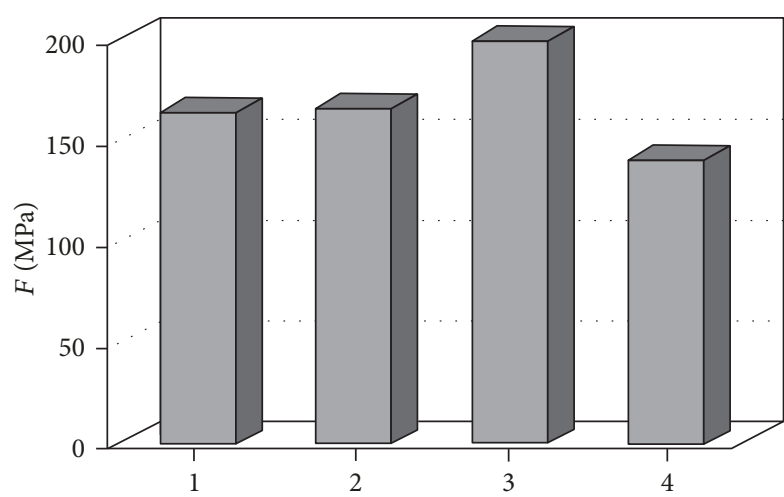

(a)

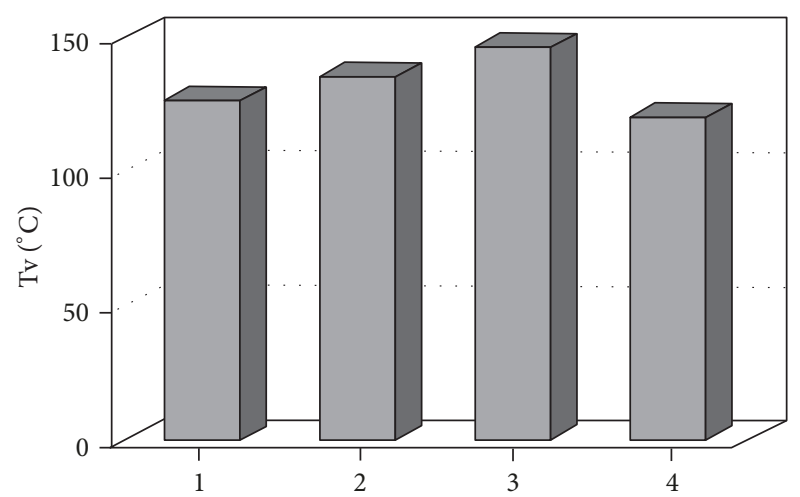

(b)

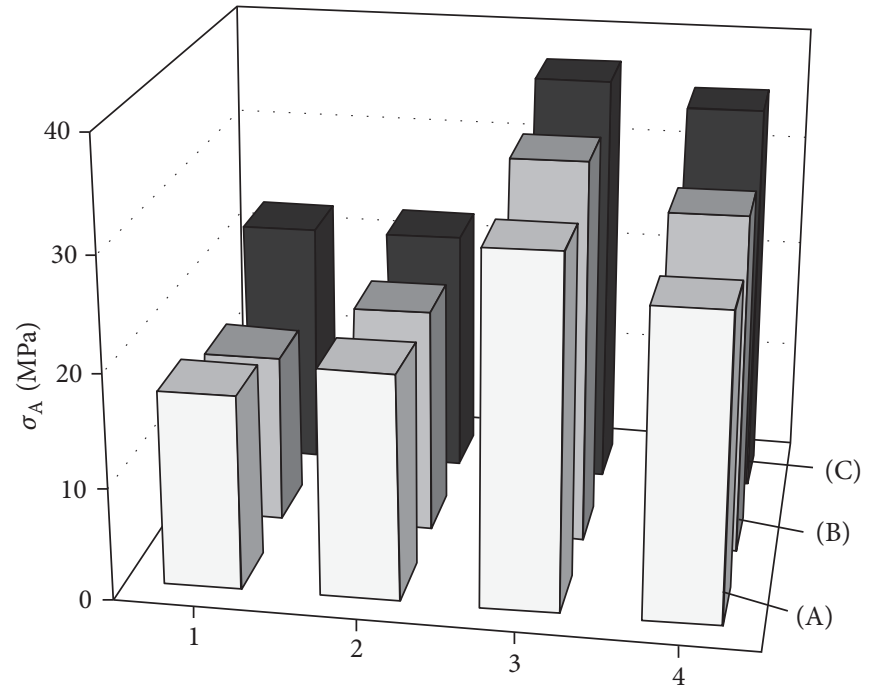

(c)

FIGURE 8: Surface hardness (a), Vicat softening temperature (b), and the strength of adhesive bonding (c) MMA-PVP-gr-PMMA compositions depending on comonomer nature: 1, without comonomer; 2, VP; 3, GMA; 4, HEMA. Nature of support material: (A) steel/steel; (B) ceramics/ceramics; (C) steel/PMMA.

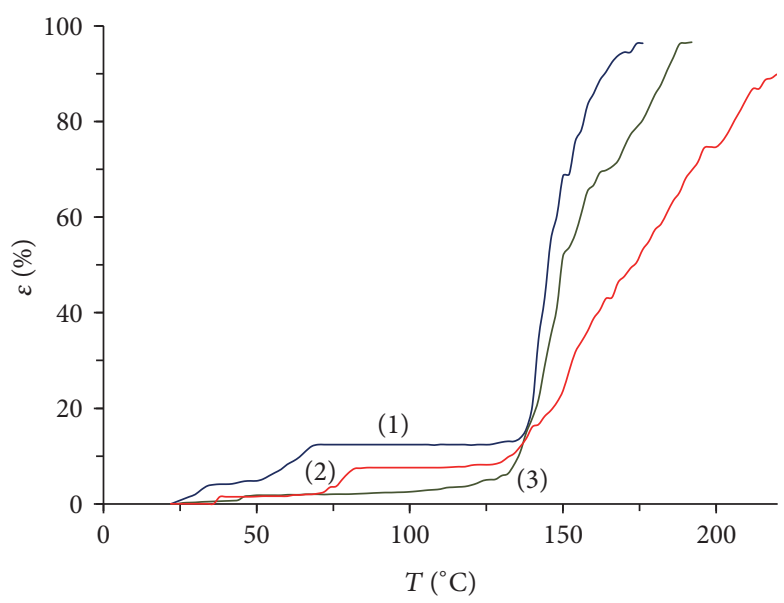

FIGURE 9: Thermomechanical curves of materials based on MMAPVP-gr-PMMA compositions: (1), (3) not heat-treated, (2) heattreated at $333 \mathrm{~K}$; (3) with $\mathrm{SiO}_{2}$. Filler content: 10 wt.\%.
The developed materials can be used in various industries as compounds and adhesives and also in dentistry as components of filling materials, toning lacquers, and protective coatings.

\section{Conflicts of Interest}

The authors declare that they have no conflicts of interest.

\section{References}

[1] L. M. Al-Harbi, S. A. Kosa, M. K. Baloch, Q. A. Bhatti, and E.S. E.-B. H. El-Mossalamy, "Adsorption of Polyvinylpyrrolidone over the Silica Surface: As Affected by Pretreatment of Adsorbent and Molar Mass of Polymer Adsorbate," International Journal of Polymer Science, vol. 2016, Article ID 2417292, 2016.

[2] J. W. Sikora, V. Levytskyi, V. Moravskyi, and H. Gerlach, "Twin screw extrusion with Expancel foaming agent," Journal of Polymer Engineering, vol. 33, no. 6, pp. 501-508, 2013. 
[3] V. E. Levyts'kyi, A. S. Masyuk, D. S. Samoilyuk, L. M. Bilyi, and T. V. Humenets'kyi, "Morphology and Properties of Polymer-Silicate Composites," Journal of Materials Science, vol. 52, no. 1, pp. 17-24, 2016.

[4] V. Levytskyi, A. Masyuk, D. Katruk, and M. Bratychak, "Regularities of obtaining, morphology and properties of metalcontaining polymer-silicate materials and polyester composites on their basis," Chemistry and Chemical Technology, vol. 10, no. 1, pp. 35-40, 2016.

[5] V. Skorokhoda, Y. Melnyk, V. Shalata, T. Skorokhoda, and S. Suberliak, "An investigation of obtaining patterns, structure and diffusion properties of biomedical purpose hydrogel membranes," EasternEuropean Journal of Enterprise Technologies, vol. 1, no. 6-85, pp. 50-55, 2017.

[6] S. P. Davtyan, A. O. Tonoyan, A. Z. Varderesyan, and S. C. Müller, "Frontal copolymerization in the presence of nanoparticles," European Polymer Journal, vol. 57, pp. 182-186, 2014.

[7] N. M. B. Smeets, "Amphiphilic hyperbranched polymers from the copolymerization of a vinyl and divinyl monomer: The potential of catalytic chain transfer polymerization," European Polymer Journal, vol. 49, no. 9, pp. 2528-2544, 2013.

[8] A. M. Oliveira, K. L. Guimarães, and N. N. P. Cerize, “The role of functional monomers on producing nanostructured lattices obtained by surfactant-free emulsion polymerization - A novel approach," European Polymer Journal, vol. 71, article no. 7015, pp. 268-278, 2015.

[9] P. Klemarczyk and J. Guthrie, "Advances in anaerobic and cyanoacrylate adhesives," Advances in Structural Adhesive Bonding, pp. 96-131, 2010.

[10] H. Kilambi, S. K. Reddy, L. Schneidewind, J. W. Stansbury, and C. N. Bowman, "Copolymerization and dark polymerization studies for photopolymerization of novel acrylic monomers," Polymer Journal, vol. 48, no. 7, pp. 2014-2021, 2007.

[11] L. Liu, W. Feng, X. Lü, and W.-K. Wong, "First example of near-infrared (NIR) luminescent $\mathrm{Yb} 4$ (Salen)4-containing metallopolymer through radical copolymerization with MMA (methyl methacrylate)," Inorganic Chemistry Communications, vol. 75, pp. 29-32, 2017.

[12] S. Pal, B. Banoth, G. Rahithya, A. Dhawan, and P. De, "Copolyperoxides of 2-(acetoacetoxy)ethyl methacrylate with methyl methacrylate and styrene; Synthesis, characterization, thermal analysis, and reactivity ratios," Polymer (United Kingdom), vol. 53, no. 13, pp. 2583-2590, 2012.

[13] H. Lutz, H.-P. Weitzel, and W. Huster, "Aqueous Emulsion Polymers," Polymer Science: A Comprehensive Reference, 10 Volume Set, vol. 10, pp. 479-518, 2012.

[14] X. Liu, Z. Wu, F. Zhou, D. Li, and H. Chen, "Poly(vinylpyrrolidone-b-styrene) block copolymers tethered surfaces for protein adsorption and cell adhesion regulation," Colloids and Surfaces B: Biointerfaces, vol. 79, no. 2, pp. 452-459, 2010.

[15] K. Shen, D. Qi, Y. Li, X. Zhao, and Y. Li, "Controlled emulsion polymerization of styrene and methylmethacrylate in the presence of amphiphilic tertiary amine N-oxides," Colloids and Surfaces A: Physicochemical and Engineering Aspects, vol. 482, pp. 728-733, 2015. 


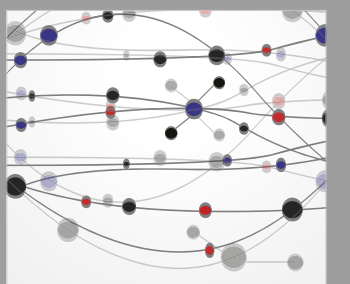

The Scientific World Journal
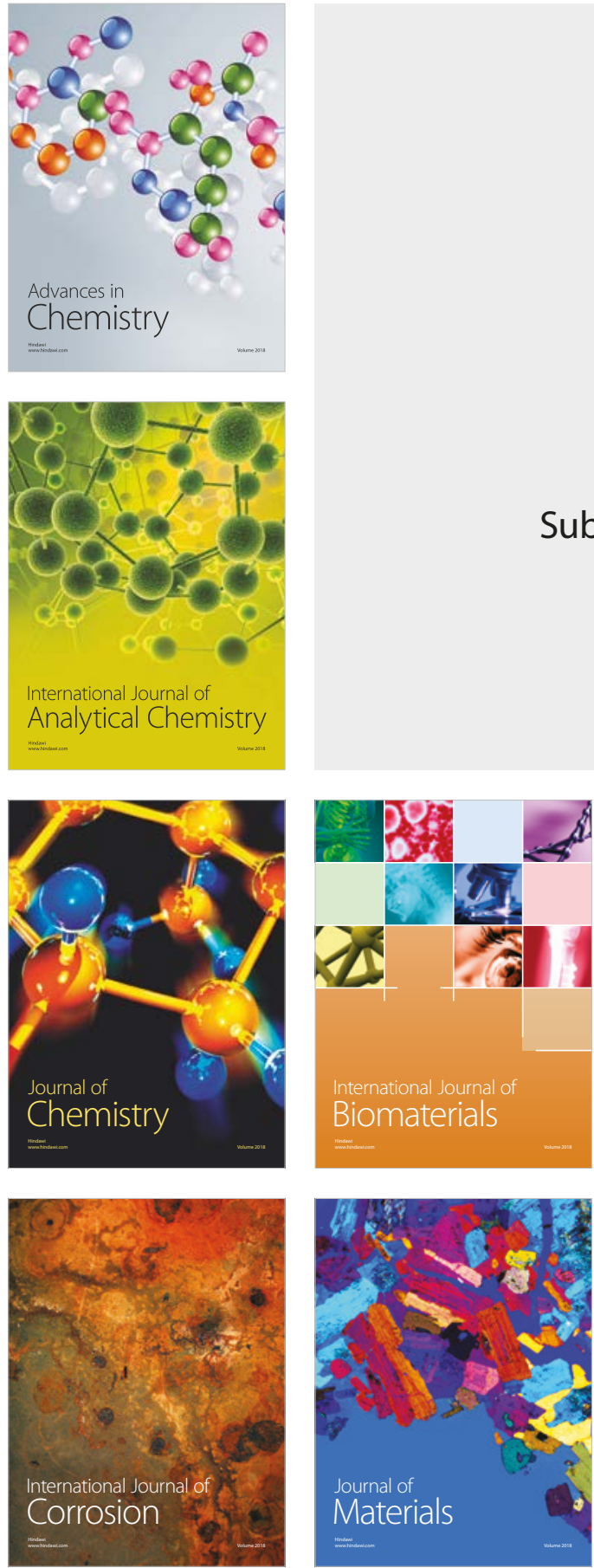

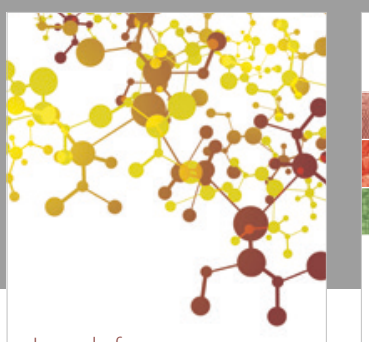

Journal of

Applied Chemistry
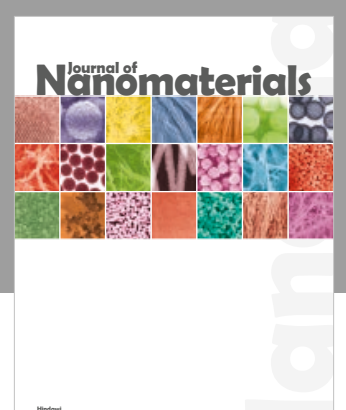

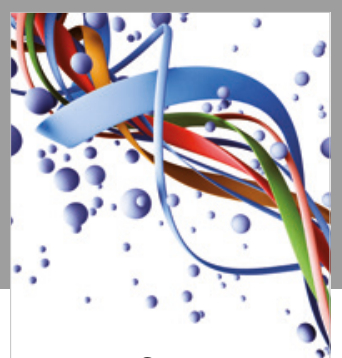

Scientifica

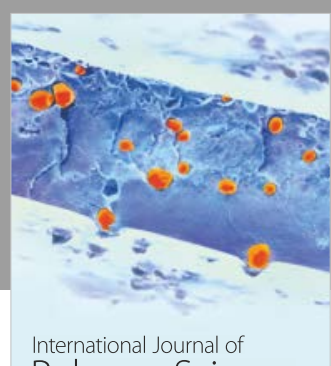

Polymer Science

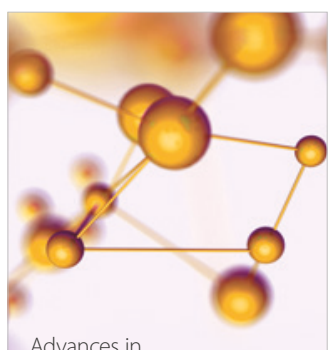

Physical Chemistry
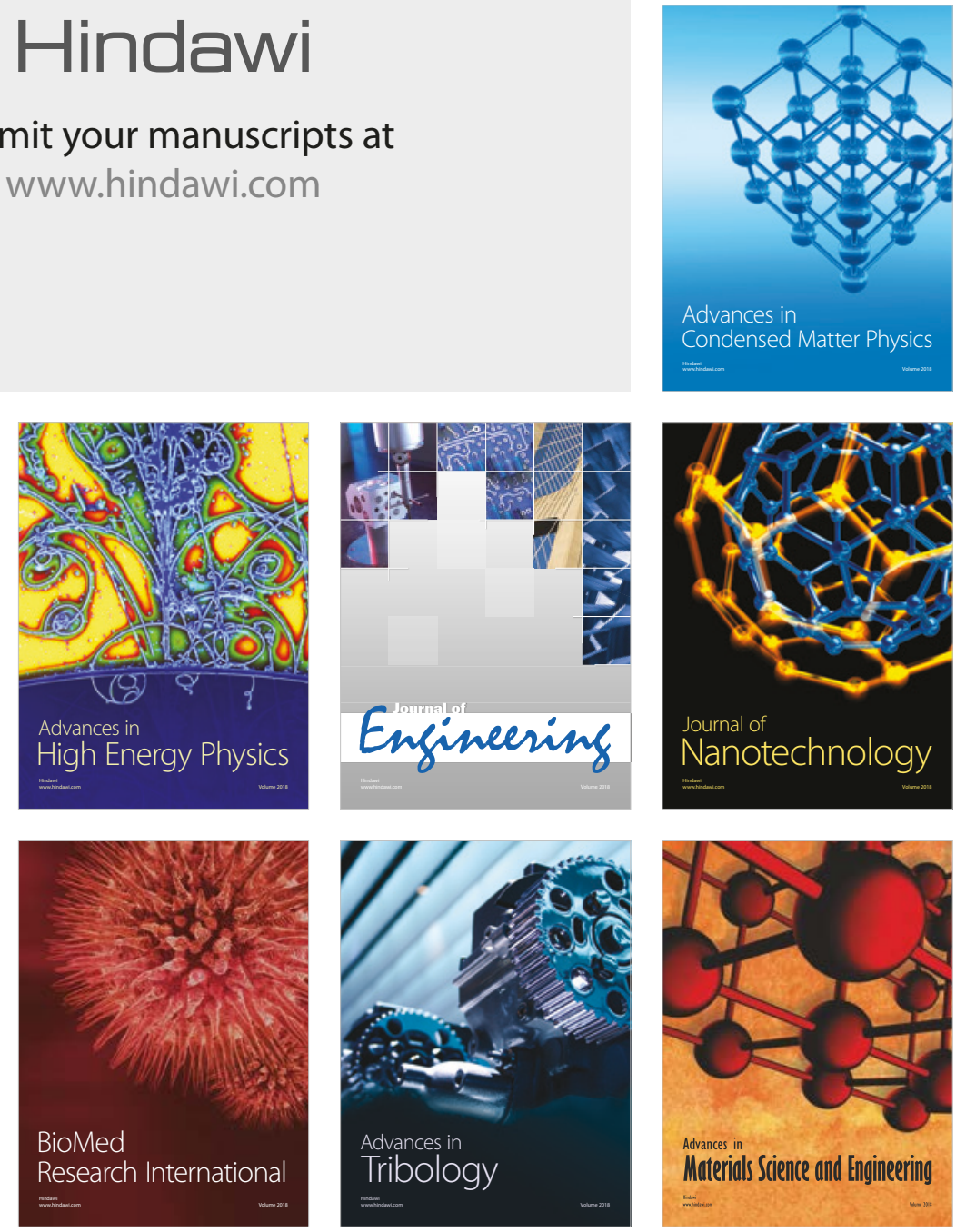